\title{
Inhaltsverzeichnis
}

Vorwort . . . . . . . . . . . . . . . . . . XIII

\section{KAPITE L}

Probleme der Rezeptionsgeschichte der Barockliteratur. Daniel Casper von Lohenstein und sein Publikum

Inhalt: Die Entdeckung des Lesers und die Entstehung der Rezeptionsästhetik, S. I Der Versuch der neuen Ästhetik, zwischen Literatur und Geschichte, historischer und ästhetischer Erkenntnis zu vermitteln, S. 3 - Rezeptionsästhetik und Literatursoziologie, S. 4 - Problematik der Wolffschen Theorie des »idealen Lesers«, S. 5 - Die Thesen Wolfgang Isers und die Reduktion des Lesers auf eine reine Formalkategorie, auf ein "Kompositionselement« des literarischen Textes, S. 6 - Der tschechische Strukturalismus und die Entdedkung des Lesers als eines integrierenden Bestandteils der Struktur des literarischen Textes, S. 9 - Die Kenntnis des Literaturwerks als Kenntnis der »Geschichte seiner unzählige Male wiederholten Lektüre« (Arthur Nisin), S. Io - Mukařovskýs Unterscheidung zwischen »Artefakt « und »ästhetischem Gegenstand «, S. I I - Felix Vodičkas Begriff »Konkretisation«, S. I2 - Mukařovský und die Rekonstruktion des für ein bestimmtes Publikum verbindlichen Systems von "ästhetischen Normen «, S. $x_{3}$ Kodifizierte und nichtkodifizierte Norm, S. I 3 - Mukařovskýs Begriff »Evolutionswert« und die Theorie der literarischen Evolution bei den russischen Formalisten, S. Is - Hans Robert Jauß und sein Versuch, den ästhetischen Wert des Literaturwerks zu quantifizieren, S. 16 - Der Begriff »Erwartungshorizont «, S. 16 - Die Rekonstruktion der "Erwartungshorizonte», S. 18 - Unannehmbarkeit des Vorschlags von Jauß, den ästhetischen Wert des Literaturwerks auf Grund der Distanz zwischen diesem und dem Erwartungshorizont des Publikums zu bestimmen, S. 19 - Gründe für die Grenzen von Jaußens ästhetischer Betrachtung. Soziologische Undifferenziertheit der Begriffe Publikum und Erwartungshorizont bei Jauß. Auffassung der Entwicklung der »literarischen Reihe a als autonom und immanent, S. 22 - Das Problem der Neuerung und der Automatisierung von Formalmustern und die Beziehung zwischen Stabilität der Sozialstruktur und Evolution künstlerischer Formen, S. 23 - Die Koexistenz verschiedener Gruppen innerhalb des literarischen Publikums sowie verschiedener ästhetischer Normen und die strukturelle Öbereinstimmung zwischen Normenhierarchie und Gesellschaft, S. 23 - Die Notwendigkeit einer beständigen empirischen Uberprüfbarkeit der Grundsätze der Rezeptionsästhetik, S. 24 - Die Auffassung der Literatur als eines Sondersystems sozialer Kommunikation und ihre Eignung zur Vertiefung der Beziehungen zwischen Rezeptionsästhetik und Geschichte, S. 25 - Der Begriff des ästhetischen Kodes, S. 26 - Richtige und falsche Dekodierungen, S. 27 - Kunstbetrachtung im Umkreis von Kommunikationsvorgängen und die Lösung des Problems des ästhetischen Vergnügens, S. 27 - Homogenität des deutschen literarischen Publikums des 17. Jahrhunderts, S. 28 - Die Gelehrten: Produzenten und Konsumenten des Buchmarkts, S. 29 - Die deutsche Buchproduktion in der zweiten Hälfte des 17. Jahrhunderts, S. 3 I - Buchproduktion und Buchrezeption als typisch elitäre Erscheinungen, S. 34 - Die Buchproduk- 
tion in lateinischer Sprache in Deutschland und Frankreich, S. 34 - Zahlenmäßige Konsistenz des literarischen Publikums des 17. Jahrhunderts, S. 35 - Wandel in der Buchproduktion des 18 . Jahrhunderts, S. 36 - Literarisches Publikum und Universitätsbesuch, S. 37 - Desinteresse der Mittelschicht am Buch, S. 38 - Geringe Produktion von Dichtwerken, S. 39 - Das Publikum der Dichtwerke, S. 39 - Die Produktion von Dichtwerken in Deutschland und England, S. 39 - Beständige Abnahme der theologischen Buchproduktion und gleichzeitige Zunahme der dichterischen Produktion im Verlauf des 18. Jahrhunderts, S. 40 - Bibliophiler Eifer im 17. Jahrhundert, S. 42 Die übliche Lektüre der Mittelklassen, der unteren Volksschichten und des Bauernstandes. S. 42 - Die Adelsschicht als "Träger « der Dichtung. Die Bibliotheken der Adeligen, S. 45 - Die Produktion der Romanliteratur im 17. Jahrhundert, S. 54 - Die Preise der höfisch-historischen Romane, S. 56 - Zahlenmäßige Konsistenz des Publikums der dichterischen Produktion, S. 60 - Der Gelehrtenstand im 16. und 17. Jahrhundert, S. 6 I Entstehung der Hofbürokratie und Rezeption des römischen Rechts in Deutschland, S. 66 - Universitätsbesuch des Adels im 16. und 17. Jahrhundert, S.69 - Die Vertretung des Adels und des Bürgertums in den zentralen Regierungsorganen der Territorien und des Reiches, S. 73 - Die gesellschaftliche Integration zwischen adeliger und bürgerlicher Beamtenschaft im reaktiven Milieu des Hofes im Verlauf des 17. Jahrhunderts, S. 77 - Sozialer und geistiger Zustand der bürgerlichen Intelligenz, S. 78 - Entstehung der aristokratisch-höfischen Kultur aus der Amalgamierung zwischen adeligfeudalem und bürgerlich-städtischem Element, S. 80 - Die Höflichkeit, S. 8 I - Notwendigkeit des Besuchs von Höfen sowohl für den Beamten eines Fürsten als auch für den Dichter, S. 83 - Hof und Repräsentation, S. 86 - Die Barodkdichtung als Repräsentation der Macht, S. 88 - Die höfischen Werte, S. 89 - Der Gymnasialunterricht im 16. und 17. Jahrhundert, S. 95 - Primat der Rhetorik im Gymnasial- und Universitätsunterricht, S. 99 - Die Barodkisierung der Rhetorik durch die Jesuiten, S. 100 Die Gründung der »Ritterakademien* und ihr pädagogisches Ideal, S. ros - Der Beitrag der Sprachgesellschaften zum kulturellen Integrationsprozeß zwischen Adel und Bürgertum, S. I09 - Die Funktion der Sprachgesellschaften als Zentren der literarischen Kultur in Deutschland, S. II I - Fehlen einer literarischen Hauptstadt im deutschen Sprachgebiet des 17. Jahrhunderts, S. I I I - Die deutschen Literaturzentren des 17. Jahrhunderts, S. I Is - Die adeligen Schriftsteller Niederösterreichs und ihre literarischen Beziehungen zu Weimar und Nürnberg, S. I 6 - Stubenbergs Tärigkeit als Übersetzer italienischer und französischer Barodkromane, S. I17 - Die Theorie des geschichtlichhöfischen Barodkromans, S. I I9 - Der Adel als Adressat des geschichtlich-höfischen Romans, S. I 20 - Die gesellschaftliche Funktion des Romans: Unterweisung in der Höflichkeit, in der höfischen Beredsamkeit, in der politischen Doktrin und den Adelstugenden sowie Vermittlung einer allumfassenden Bildung, S. I20 - Labyrinthische Struktur des Romans, S. r 24 - Der Roman als Theodizee und als »Fürstenspiegel«, S. I 27 Die Entschlüsselung der Hinweise und Anspielungen auf Personen und Aktuelles sowie die Erkenntnis der geometrischen Struktur des Romans als Quellen eines ästhetischintellektuellen Vergnügens für Leser, die den ästhetischen und kulturellen Kode des Autors besitzen, S. I29 - Zugehörigkeit des geschichtlich-höfischen Barockromans zum Kommunikationsbereich der "repräsentativen Offentlichkeit", S. I3 I - Die Theorie der Tragödie im Barock, S. I 3 I - Die Tragödie als politische Schule, S. I32 - Die ethische Funktion der Tragödie, S. I 33 - Die Idealisierung der tragischen und epischen Helden, S. 133 - Der barocke Mensch und der Verlust des Individualitätsgefühls, S. I 34 - Die 
Welt als Theater, S. I35 - Die Entstehung des metaphorischen Kodes der Barodkdichtung aus dem Bewußtsein der Relativität der Wirklichkeit, S. 137 - Die Funktion der Dichtung gemäß der barocken Ästhetik des Scharfsinns, S. I 39 - Die Emblematik, S. 140 - Notwendigkeit der Kenntnis des emblematischen Kodes zur Entschlüsselung der Barockdidhtung, S. 143 - Das barodke Ideal der Polymathie und sein Ursprung, S. 144 - Schriftsteller und literarisches Publikum im 17. Jahrhundert. Ihre kulturelle und gesellschaftliche Homogenität, S. I46 - Ausschlaggebender Beitrag der Beamten der höfischen und der städtischen Bürokratie sowie des Adels zur Literatur und Poesie des 17. Jahrhunderts, S. I48 - Die enge Beziehung der Barockdichtung zur Macht und zu den herrschenden Klassen, S. 148 - Fürsten, Adel und Patriziat als Adressaten der hohen Barockdichtung, aufgefaßt als "repräsentative* ästhetische Kommunikation, S. 149 - Lohenstein und sein Publikum, S. I53 - Die kulturelle Homogenität zwischen Dichter und Publikum, die im Hof ihr gemeinsames geistiges Zentrum haben. Dieses bewirkt die Entsprechung zwischen den Werken des ersteren und dem Erwartungshorizont des letzteren, S. 170 - Das rhetorische und repräsentative Wesen der Barodkdichtung als Ausdruck jener tiefen geistigen Integration zwischen Autor und Publikum, den beiden Polen der ästhetischen Kommunikation, die in der weitgehenden sozialen Integration zwischen der intellektuellen und der führenden Schicht gründet (dies wird durch die für das 17. Jahrhundert charakteristische gesteigerte soziale Mobilität ermöglicht), S. I72.

\section{K A PIT'E L}

Die barocke Apotheose. Der unvergleichliche Lohenstein (I66I-I 73 I)

Inhalt: Die ersten Dokumente zur Rezeptionsgeschichte Lohensteins. Die Periochen zu Cleopatra, Agrippina, Epicharis und Sophonisbe, S. 175 - Die Aufführung des Ibrabim Bassa in Danzig, S. 177 - Das Vorwort von Kormart zur Ubersetzung der Maria Stuart von Vondel, S. 178 - Birkens Teutsche Dicht-Kunst, S. 178 - Hoffmannswaldaus Vorwort zu seinen Deutschen Ubersetzungen, S. 179 - Morhofs Unterricht, S. 179 Kretschmers Devoti charakter animi, S. 180 - Die Poëseos Germaniae bistoria von Chenius, S. 18r - Der Ursprung von Lohensteins Ruhm und die Struktur der gelehrten und literarischen Information im I7. Jahrhundert, S. 182 - Die Trauergedichte auf Lohensteins Tod, S. 185 - Der Lebens-Lauff von Hans Casper von Lohenstein, S. I 87 Die Vollständige Deutsche Poesie von Rotth, S. 189 - Die ersten Publikationsankündigungen des Arminius im Catalogus Universalis, S. 190 - Die Rezension des Arminius in den Acta Eruditorum, S. 19I - Die Rezension von Thomasius, S. 197 - Die Rezension von Tentzel, S. 203 - Die Anmerkungen von Christian Wagner, S. 204 - Neukirchs Vorwort zum Arminius, S. 212 - Die dem Roman vorangestellten Widmungsgedichte, S. 216 - Christian Weises Analyse von Lohensteins Stil, S. 218 - Neumeisters Specimen, S. 22 I - J. G. Meisters Unvorgreiffliche Gedancken Von Teutschen Epigrammatibus, S. 223 - Heideggers Mythoscopia Romantica, S. 224 - Werenfels' Dissertatio de meteoris orationis, S. 228 - Grob und seine Polemik gegen den Barodkroman, S. 230 Die Rezensionen zur Mythoscopia Romantica von Leibnitz-Edkhart und von Gundling, S. 23 I - Wernickes antibarodke Polemik, S. 234 - Die literarische Fehde zwischen Wernicke und Hunold, S. 239 - Schröters »lohensteinische « Rhetorik, S. 242 - Männlings Auszüge, S. 246 - Die Ausgabe des Arminius von I73r, S. 253 - Breslers De vita et scriptis Danielis Caspari à Lohenstein, S. 264 - Die Darstellung Lohensteins und seines dichterischen Werkes in der Überlieferung von Enzyklopädien und verschiedenen 
gelehrten Schriften, S. 265 - Albrecht von Haller, S. 279 - Johann Christian Günther, S. 280 - Benjamin Neukirch und sein Abrüdken von der schlesischen Barodkdichtung, S. 283 - Gesellschaftliche Ursachen der Entwidklung des literarischen Geschmadks im antibarodken Sinn, S. 286 - Gründe für Lohensteins Erfolg zwischen r66I und I73 I, S. 287.

\section{K A P ITE L}

Die Kritik der Aufklärung. "Die Lohensteinische schwülstige Schreibart (1722I 800)

Inhalt: Die ersten Rhetoriken der Aufklärung und die Ablehnung der spezifischen Elemente des stilistischen und rhetorischen Kodes des Barodk, S. 291 - Das Ideal der $\# \mathrm{Na}$ türlichkeit火 in der moralischen Literatur und in den Anstandsbüchern, S. 295 - Der Wandel in der Poetik und der Literaturkritik in den ersten Jahrzehnten des I 8. Jahrhunderts, S. 296 - Johann Ulrich Königs Untersuchung von dem guten Geschmack, S. 298 - Jundkers Untersuchung der Hanckischen Gedichte, S. 299 - Handkes Poetischer Staar-Stecher, S. 300 - Die Polemik gegen das Barodk und gegen Lohenstein in den Discoursen der Mablern, S. $30 \mathrm{I}$ - Die von den Moralischen Wochenschriften propagierten stilistischen und ethischen Ideale, S. $30_{3}$ - Der Lektürekanon der Moralischen Wochenschriften der ersten Hälfte des i 8. Jahrhundert, S. 3 I I - Der Mabler der Sitten, S. 3 I 4 - Von dem Einflu $\beta$ und Gebrauche der Einbildungs-Krafft von Bodmer und Breitinger, S. 3 I9 - Bodmers Vergleichung zwischen Lobensteins Arminius und Heideggers Apollo Auricomus, S. 322 - Ideologische Motive für Bodmers Polemik gegen Lohenstein, S. 323 - Die Critische Abhandlung von der Natur der Gleichnisse und Breitingers Kritik an Metapher und Gleichnis im Barock, S. 326 - Jakob Immanuel Pyra und sein Erweis, S. 335 - Die Kritik Gottscheds, S. 337 - Gottscheds Analyse des Panegyrikus zum Tode Hoffmannswaldau als exemplarisches Muster für die aufklärerische Methode, die Barockdichtung zu diffamieren, S. 34I - Der Anti-Longin von Johann Joachim Schwabe, S. 350 - Die Critische Untersuchung von Mylius, S. 352 - Der Critische Musikus von Scheibe und der Vergleich Bach-Lohenstein, S. 355 - Folgen der totalen Konfrontation zwischen klassizistischem und barodkem Stil, S. 357 - Der Lektürekanon in Gottscheds Lehrbüchern für den Gymnasialunterricht, S. 358 - Die Polemik Gottscheds und seiner Schüler gegen die »lohensteinische " Dichtung Klopstodks, S. 359 - Die Abhandlung von den Gleichnissen von Curtius, S. $3^{65}$ - Die Abhandlung von andern Tragödien, die auch von Sophonisben bandeln von Johann Heinrich Schlegel, S. 366 - Erneute Schätzung Lohensteins im Historischen Entwurf von den Verdiensten der Evangelischen Gymnasiorum in Breßlau um die deutsche Schaubübne von Johann Caspar Arletius, S. 369 - Mendelssohns Apologie von Lohensteins Prosastil, S. 369 - Wirkung der Rehabilitierung des Stils des Arminius durch Mendelssohn auf die Literaturkritik und -geschichtsschreibung, S. 370 - Friedrich Justus Riedels Briefe über das Publikum: erster Versuch einer historischen Interpretation von Lohensteins Dichtung, S. 375 - Das Urteil über Lohenstein in Christian Heinrich Schmids Kompilationen, S. $37^{8}$ - Die Abhandlung Lobenstein, als dramatischer Dichter von Heinrich Daniel Zschokke und seine "Ehrenrettung " des schlesischen Dichters, S. ${ }_{3}^{81}$ Das Bild Lohensteins in historischen Darstellungen der Literatur, der Gelehrsamkeit und des Theaters aus der zweiten Hälfte des 18 . Jahrhunderts, S. 385 - Unmöglidkeit einer richtigen Dekodierung von Lohensteins Dichtung wegen des radikalen Wandels der ästhetischen Normen im Verlauf des I8. Jahrhunderts, S. 396 - Auswirkungen der 
radikalen Veränderung des literarischen Geschmadks auf die Buchproduktion des I 8. Jahrhunderts. Auffallender Rückgang der emblematischen Literatur; Ursachen und Folgen, S. $400-$ Der in den Schulanthologien des I 8. Jahrhunderts vorgeschlagene Lektürekanon und sein Einfluß auf die literarische Geschmadksbildung, S. 401 - Gellerts `Klassizität in der zweiten Hälfte des I8. Jahrhunderts, S. 404 - Der von den Moralischen Wochenschriften in der zweiten Hälfte des 18 . Jahrhunderts propagierte Lesekanon und ihr starker Einfluß auf die Geschmacksbildung beim literarischen Publikum, S. 4II - Lesegesellschaften und Leihbibliotheken. Weitere Verbreitung des Buches und fortschreitende Kommerzialisierung des literarischen Lebens, S. 413 - Tiefgreifende strukturelle Veränderungen in der Buchproduktion zwischen 1740 und 1800 . Explosionsartige Ausbreitung der Belletristik und gleichzeitiger Rückgang der theologischen und der Erbauungsliteratur, S. 4I4 - Zunahme des literarischen Publikums und dementsprechende Entwidklung eines ausgedehnten Buchmarktes, S. $4_{16}$ - Soziale und kulturelle Heterogenität des literarischen Publikums der zweiten Hälfte des I8. Jahrhunderts, S. 417 - Der Prozeß der >Verbürgerlichunge der Literatur und ihre Ursachen, S. 419 Das Problem der literarischen Intelligenz des I 8. Jahrhunderts. Fortschreitende Abnahme der sozialen Mobilität und >Verbürgerlichung، der Intellektuellenschicht, S. 4 I9 - Verlust an sozialem Prestige und von Machtpositionen in den zentralen Regierungsorganen der deutschen Territorien und des Reiches seitens der bürgerlichen Intelligenz im Verlauf des 18. Jahrhunderts, S. 423 - Hegemonie des Uradels in den hohen Rängen der Territorial- und der Reichsbürokratie und gleichzeitiges Zurückweichen des Bürgertums in der zweiten Hälfte des I 8. Jahrhunderts, S. 424 - Gotha und Hannover als Extremfälle der Unterbrechung des Osmose-Prozesses zwischen bürgerlicher Intelligenz und Adel, S. 423 - Ständige Verringerung des Index der vertikalen Mobilität im Heer, S. 425 - Umwandlung des Patriziats der Freien Reichsstädte in eine geschlossene Klasse, S. 425 - Ende des Prozesses der sozialen Integration von politischer und intellektueller Klasse und genetische Verknüpfung zwischen der Verdrängung der bürgerlichen Intelligenz in eine soziale Randposition und der politischen, sozialen und ästhetisch-literarischen Ideologie der Aufklärung, S. 426 - Gründe für die Verringerung der sozialen Mobilität. Schrumpfung der Bürokratie. Aristokratisierung des absolutistischen Staates in der zweiten Phase seiner Entwidklung. Aristokratisierung des Adels und dessen Ubernahme französischer Sitten, Sprache und Kultur, S. 427 - Vom absolutistischen Staat gefördertes Bündnis zwischen Intellektuellenschicht und Bürgertum, S. 429 - Allianz der Hohenzollern mit dem Pietismus, S. 430 - Die Gesellschaftstheorie des Pietismus und ihr Beitrag zum Abbau des Feudalismus und zur Umwandlung der deutschen Gesellschaft in eine bürgerliche Gesellschaft, S. 43 I - Herkunftsschicht der Begründer und der Anhänger des Pietismus, S. 43 I - Preußen als Zentrum der antibarocken literarischen Kultur, S. 432 - Indirekter und direkter Beitrag des Pietismus zur Überwindung und Ablehnung der Barodkdichtung, S. 433 - Die Phasenverschiebung im Entwicklungsprozeß des Barock zwischen der Literatur einerseits und den darstellenden Künsten sowie der Musik andrerseits stellt die Gegenprobe dazu dar, daß die Abkehr der bürgerlichen literarischen Intelligenz von den Formen der barocken Repräsentation des Absolutismus hauptsächlich wegen der verminderten Geschwindigkeit der Zirkulation der Eliten erfolgt, S. 434 .

Personenregister . . . . . . . . . . . . . . 437 Tafeln . . . . . . . . . . . . . . . $\operatorname{nach~} 458$ 
